\section{Biological properties of immunogiobulins}

Comprehensive Immunology. Volume 5: Immunoglobulins. Edited by $\mathrm{R}$. A. Good and G. W. Litman. Pp. 381. (Plenum Medical: New York and London, 1978.) \$18.58.

ThIS is a multi-authored book containing 16 different articles. The style of each is governed more by the authors themselves than by any unifying editorial criterion. The length of the chapters is also very variable and bears no relationship to their general importance. Whether this is by chance or design, it turns out to be fortunate because, in general, the most extensively, and to my mind the better, reviewed subjects have also suffered the least from the unavoidable delay in publication of books of this nature.

The major emphasis is on the more classical immunochemistry problems concerned with the nature of the antibody-antigen interaction. Crystallographic, physicochemical, kinetic and thermodynamic data bring us a comprehensive picture of the general properties of the antibody-combining sites and, more importantly, of the outstanding and unresolved aspects of the problem. For those of us trying to understand the peculiarities of monoclonal antibodies in serological reactions, some of the chapters (particularly that by Karush on "The Affinity of Antibody: Range, Variability, and the Role of Multivalence") are very relevant. Yes, Cathou was right in saying, "Immunochemistry does not appear to be in any danger of an early death" (p76).

The first six chapters, which also include discussions on the structural basis of the effector functions of antibodies and other biological properties, take up well over half of the book. The other portion doesn't attempt a comprehensive coverage of other aspects of immunoglobulins, but a quick glance at the table of contents may give a misleading impression of the real subject matter. For instance, the short discussion by Ohno on the significance of gene duplication in Ig evolution is intended for the initiated and not to give a picture of the complexities of the contraction and expansion processes in the evolution of immunoglobulin gene pools. On the other hand, I found a considerable amount of information on subjects that are seldom reviewed: for example, on immunoglobulin-like molecules and possible functional ancestors of antibodies in lower species (chapter 8) and on the relative expression of $V_{H}$ subgroups in normal and abnormal immunoglobulins in man (chapter 13).

Chapter 10 reviews the structure of atypical immunoglobulins and their implications in terms of genetic control mechanisms. It is a great pity that Frangione did not have an extra year to write this chapter, as his pioneering ideas about a separate 'gene' for the hinge region could have crystallised with the discoveries of 'gaps' in 'the DNA structure. The book closes with a crisp "Overview on membrane immunoglobulins": the outstanding problems, the relevant facts and the prevailing opinions at the time of publication. Pernis has obviously made a great effort to try to include last-minute references and, in the rush, Berget et al. (1977) did not make it to the reference list.

A number of errors have crept into

\section{Field theory}

Relativistic Quantum Fields. By C. Nash. Pp.223. (Academic: London, New York and San Francisco, 1978.) $£ 15 ; \$ 31$.

IN the past five years we have witnessed an astonishing renaissance of field theory in elementary particle physics. On the one hand the WeinbergSalam unified theory of weak and electromagnetic interactions has had considerable success in fitting a large variety of neutrino neutral current data; on the other hand quantum chromodynamics (QCD) predicted and explains the scaling violations observed in deep inelastic neutrino scattering. As a result it is now the conventional wisdom that these theories, or something quite like them, will eventually permit a full understanding of all of the fundamental interactions seen in nature, except perhaps gravity.

Such an understanding is still a long way off and it will come, if at all, only when our facility in using and thinking within the field theoretic formalism has advanced considerably beyond the present level. (I am thinking, for example, of the problem of quark confinement -plainly a non-perturbative effect which will need considerable technical progress to elucidate.) It is therefore a pleasure to welcome an excellent book by Charles Nash which develops some of the fundamentals of field theory with enough detail to teach the serious student how to undertake his or her own calculations. Most of the book is devoted to the $\lambda \varphi^{4}$ theory and quantum electrodynamics (QED), rather the book here and there, some of which seem worthy of mention. For instance, Wang (chapter 9) refers to Huang as "Huong", and in chapter 10 section 4.1, called "Internal Deletions", the word "deleted" at the end of the first sentence is missing (deleted?).

According to the flap of my copy, the book was intended to "privide" (sic) a foundation for the subsequent interpretation of the biological properties of immunoglobulins. It is difficult to say if it does that, but I think it is a serious contribution to the increasingly effective communication between experts talking such different languages.

César Milstein

César Milstein is Head of the Protein Chemistry Subdivision, Medical Research Council Laboratory of Molecular Biology, Cambridge, UK

than the non-abelian gauge theories (Weinberg-Salam and QCD) which launched the revolution. This is all to the good, as it permits the assimilation of unfamiliar techniques without the encumbrances of the new theories.

The first sine qua non of the new order is functional differentiation and integration, which is illustrated by the usual Gaussian integral. (Is this the only functional integral anyone has done?) Nash takes us gently through this, and even consoles us should we feel "a little perplexed or confused". "This is quite normal". Functionals on a Grassmann algebra are also considered. Next there is an extensive coverage of dimensional regularisation, a covariant and gauge invariant method for handling the infinite quantities which inevitably arise when evaluating radiative corrections in these theories. This is used to verify the Ward Identity and to compute the renormalisation constants in QED. The final chapter touches on the asymptotically free gauge theories, the Callan-Symanzik equation, and so on. However, the coverage is less full than that of the earlier material, and there is no treatment of spontaneous symmetry breaking in non-abelian theories. If these attractive theories do withstand further rigorous experimental tests, it is to be hoped that the author will be moved to write a companion volume dealing wlth these topics in the same detail. In the meantime the present book will certainly provide a lastingly useful and relatively painless introduction into a highly technical field.

D. Bailin

D. Bailin is Lecturer in Theoretical Physics at the University of Sussex, Brighton, UK. 